\title{
Prospective Evaluation of a Goal-Directed Haemostatic Therapy Protocol for Neurotrauma
}

Schorer Raoul, Garofano Najia, Pavlovic Gordana, Licker Marc

Department of Anesthesiology, University Hospital of Geneva, Switzerland

\section{Introduction}

Intracranial or neuraxial bleeding lead to catastrophic consequences. Hemostatic treatment is therefore paramount. We evaluated a goal-directed protocol for hemostatic treatment in trauma cases by comparing the predictive power of thromboelastometry (TEM) vs. standard coagulation tests (CTs).

\section{Methods}

> Monocentric prospective cohort

> Outcomes were numbers of PRBC units transfused, and CTs obtained on the first postoperative day (POD1)

- Inclusion criteria emergency surgery >60 min, and head injury or multiple trauma including head or spine with Glasgow Coma Scale (GCS) $<13$.

> CTs and TEM performed on admission and POD1.

> TEM used intraoperatively to guide hemostatic treatment.

High-bleeder and low-bleeder groups defined ( $\geq 5$ PRBC units cutoff).

- TEM and Cts indices built by predictor summation, and used in regression.

- Coagulation analyzed through linear regression, bleeding prediction used a Poisson model.

> Logistic regression based on the transfusion cutoff.

- Mean square error and residual deviance used as indices of prediction power.

\section{Results}

21 high bleeders and 71 low bleeders

- PRBC units transfused: median 2 (IQR, 04). 25 patients not transfused.

- Both TEM and CTs associated with transfusion $(p<0.01)$.

- TEM results were the best predictor of the need for transfusion

- Prediction for bleeding: nonsignificant trend $(p=0.0506)$ in favor of TEM (figure 1).

- MCF and A15 values were the best TEM predictors for both outcomes.

- Neither TEM nor initial CTs associated with perioperative death, complications, or postoperative bronchopneumonia.

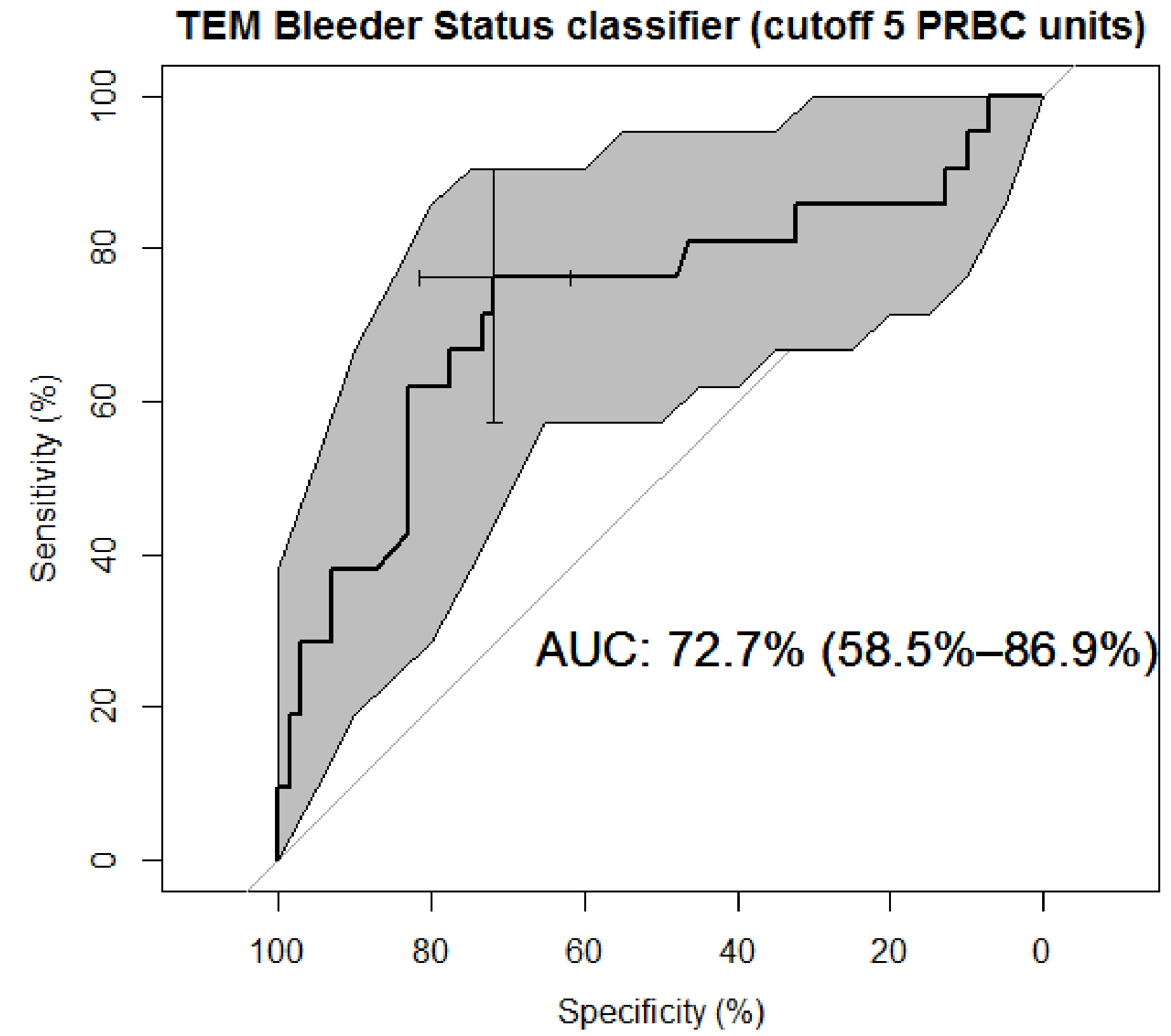

Conctusjon

Our study suggests that TEM can be used for bleeding risk stratification. More trials using TEM-based goal-directed therapy will be required to evaluate its impact on postoperative outcomes. 